\title{
Waveguide-based Detection of Protease Activity using Surface-Enhanced Raman Spectroscopy
}

\author{
Nina Turk ${ }^{1,2}$, Ali Raza ${ }^{1,2,3}$, Pieter Wuytens ${ }^{1,2,4}$, Hans Demol ${ }^{5,6}$, Michiel Van Daele ${ }^{7}$, Christophe \\ Detavernier $^{7}$, Andre Skirtach ${ }^{2,8}$, Kris Gevaert ${ }^{5,6}$ and Roel Baets ${ }^{1,2}$ \\ ${ }^{1}$ Photonics Research Group, Ghent University - IMEC, Technologiepark 126, 9052 Ghent, Belgium \\ ${ }^{2}$ Center for Nano- and Biophotonics, Ghent, Belgium \\ ${ }^{3}$ Now working at Microsoft, Espoo, Finland \\ ${ }^{4}$ Now working at IMEC, Heverlee, Belgium \\ ${ }^{5}$ VIB-UGent Center for Medical Biotechnology, Ghent, Belgium \\ ${ }^{6}$ Department of Biomolecular Medicine, Ghent University, Belgium \\ ${ }^{7}$ Department of Solid State Sciences, CoCooN Research Group, Ghent University, Belgium \\ ${ }^{8}$ Department of Biotechnology, Ghent University, Belgium \\ Authore-mail address: nina.turk@ugent.be
}

\begin{abstract}
We used a nanoplasmonic slot waveguide for the first time demonstration of waveguidebased detection of protease activity using Surface-Enhanced Raman Spectroscopy (SERS), paving the way for an integrated lab-on-a-chip protease activity monitoring.

(C) 2020 The Authors
\end{abstract}

Proteases are enzymes that catalyse the hydrolysis of peptide bonds and that are important actors in several human diseases [1]. A real-time, label-free and multiplexed method for detecting protease activity is therefore important for the development of new drugs that inhibit disease-associated proteases. In Surface-Enhanced Raman Spectroscopy (SERS), gold nanostructures can be used to enhance the specific, but weak Raman signals. Previously, SERS was shown to enable sensitive and selective detection of protease activity by monitoring cleavage of specific substrate peptides [2]. Recently, nanoplasmonic slot waveguides, shown in Figure 1(a), emerged as an alternative to the conventional Raman microscope to efficiently excite and collect SERS signals [3]. A waveguide-based SERS platform furthermore allows parallel measurements of a large number of SERS analytes, enabling high-throughput assays that are particularly of interest for pharmacological drug discovery.

Here, we demonstrate for the first time, to the best of our knowledge, waveguide-based SERS detection of protease activity using a nanoplasmonic slot waveguide, paving the way towards SERS-based monitoring of protease activity on an integrated lab-on-a-chip platform.

\section{Experiments and Results}

For our experiments, we used trypsin as a model protease. We have designed a specific peptide substrate for trypsin with the amino acid sequence $\mathrm{NH}_{2}-\mathrm{CALNN}(\mathrm{CN}-\mathrm{F}) \mathrm{GSG}(\mathrm{CN}-\mathrm{F})$ GGGGVRGNFSF-COOH, shown in Figure 1(b) [4]. The peptide contains a cysteine (C) that allows it to bind to the gold nanostructure, which provides the SERS enhancement, with the peptide forming a monolayer there. The Raman signal of the peptide comes from the two aromatic amino acids, cyano-phenylalanine (CN-F) that provides the reference SERS peak at $1180 \mathrm{~cm}^{-1}$ and phenylalanine $(\mathrm{F})$ with a peak at $1003 \mathrm{~cm}^{-1}$. Each aromatic appears in the sequence twice to double their SERS signal strength. Between the two aromatics, there is a specific cleavage site for trypsin, which cleaves C-terminal to the arginine residue $(\mathrm{R})$. After cleavage of the peptide, the short peptide fragment $\mathrm{NH}_{2}-\mathrm{GNFSF}-\mathrm{COOH}$ diffuses away from the gold surface, consequently reducing the intensity of the $1003 \mathrm{~cm}^{-1}$ peak in the SERS spectrum, as represented in Figure 1(c).
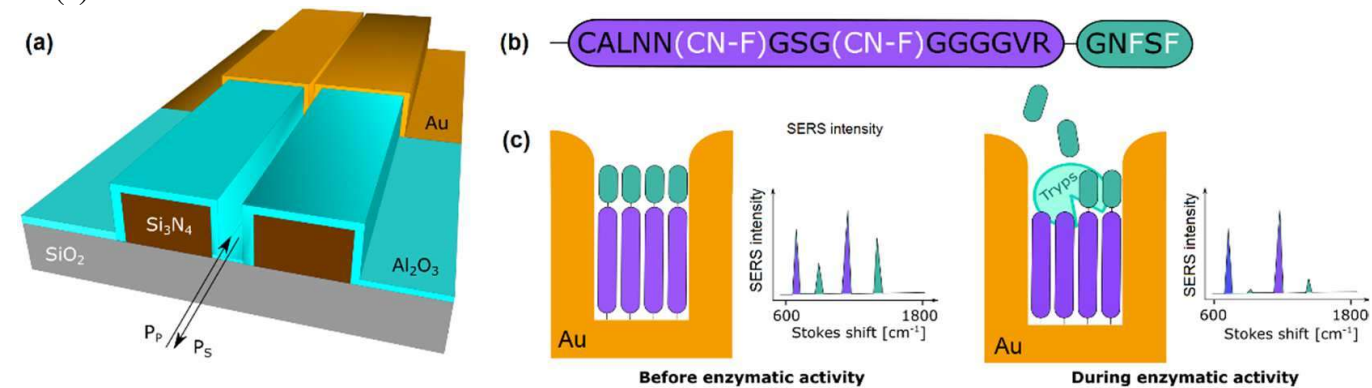

Fig. 1. (a) Nanoplasmonic slot waveguide. (b) Peptide substrate for trypsin. (c) The concept of protease activity detection via peptide cleavage using surface-enhanced Raman spectroscopy. [4] 
We performed bulk cleavage, separated the peptide fractions by RP-HPLC, labelled them on the gold nanodomes [2] and collected the SERS spectra that indeed showed the disappearance of the F peak at $1003 \mathrm{~cm}^{-1}$. We then directly labelled the nanodomes with the substrate peptide and performed trypsin cleavage directly on the peptides bound to the surface of the gold nanodomes. We noticed a $30 \%$ decrease of the F peak at $1003 \mathrm{~cm}^{-1}$, but not its complete disappearance, which can be ascribed to the limited accessibility that trypsin has to the small $12 \mathrm{~nm}$ gaps of the gold nanodomes [2].
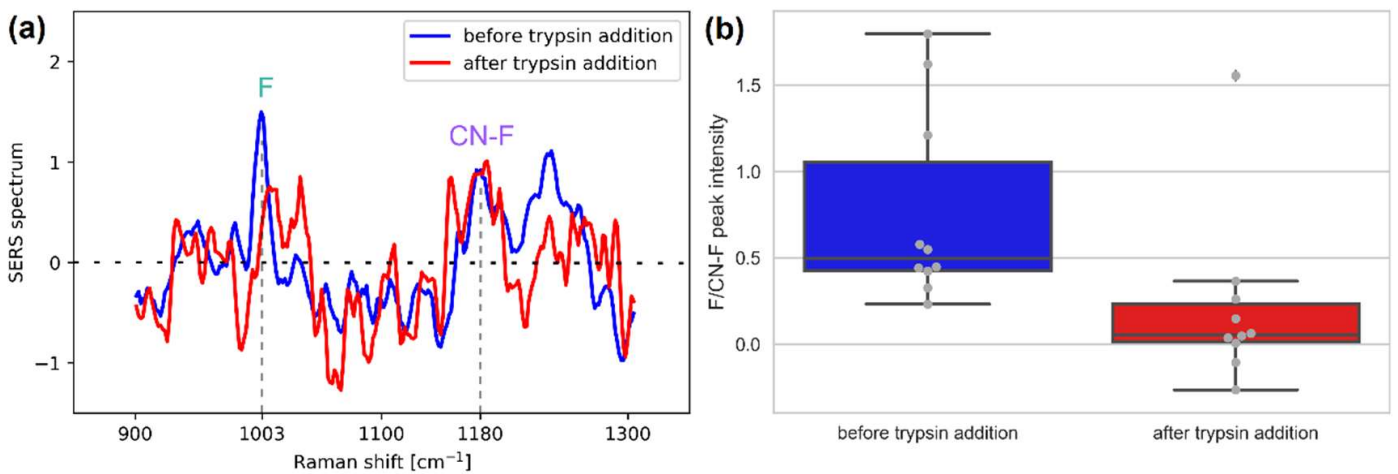

Fig. 2. (a) SERS spectra of the peptide before and after trypsin addition. The decrease in the F peak at 1003 $\mathrm{cm}^{-1}$ indicates trypsin-mediated cleavage of the peptide. Each spectrum shown in the graph is the average of 10 background-subtracted measurements. For better visualization, the spectra were smoothed with the simple moving average with the window size of 3. (b) A box plot of F/CN-F peak intensities before and after trypsin addition. Individual measurements are presented as gray dots.

Next, we labelled the peptide on the nanoplasmonic slot waveguide with the gap size of $45 \mathrm{~nm}$. The small gap width was obtained through conformal atomic layer deposition of aluminum oxide on a slotted silicon nitride waveguide made by deep UV lithography, and subsequent gold deposition [3]. We acquired SERS spectra of the peptide before and after trypsin addition using the waveguide-based excitation and collection of the SERS signal. The laser power guided in the waveguide and used to excite the SERS response was $0.3 \mathrm{~mW}$, and we recorded 10 measurements with an integration time of $60 \mathrm{~s}$ for each condition. In Figure 2(a), the averaged spectra of the background-subtracted measurements before and after trypsin addition are presented. To quantify the trypsin activity, we calculated the integrated peak counts of the $\mathrm{F}$ and $\mathrm{CN}-\mathrm{F}$ peaks at $1003 \mathrm{~cm}^{-1}$ and $1180 \mathrm{~cm}^{-1}$, respectively. To make sure that the decrease of the F peak is only caused by trypsin cleavage, we used the F/CN-F peak intensity as our metric, where the peak of CN-F serves as a normalization peak to account for potential SERS signal variation. We noticed a $70 \%$ decrease in the F/CN-F peak intensity upon one hour of trypsin incubation as shown in Figure 2(b), suggesting that trypsin has indeed cleaved the peptide substrate. We again did not notice full disappearance of the signal from the $\mathrm{F}$ peak. In this case, the remaining F/CN-F signal could be however possibly attributed to another peak that appears at $1010 \mathrm{~cm}^{-1}$ after the trypsin addition and is therefore an artefact of our data analysis. The $50 \mathrm{~nm}$ gap of the nanophotonic slot waveguide should namely allow much better accessibility to the trypsin compared to small $12 \mathrm{~nm}$ gaps of the gold nanodomes.

In conclusion, we have demonstrated for the first time ever waveguide-based SERS detection of proteolytic activity using a nanoplasmonic slot waveguide, which is the first step towards SERS monitoring of protease activity on a labon-a-chip platform. We are currently working on expanding our platform for multiplexing, in which we aim to detect the activity of two and more proteases with different specificities simultaneously.

Nina Turk acknowledges Research Foundation Flanders (FWO) for the predoctoral fellowship (1179319N).

\section{References}

[1]. M. Drag, G.S. Salvesen, "Emerging principles in protease-based drug discovery,” Nature Reviews Drug Discovery, 9, 690-701 (2010) [2] P.C. Wuytens, H. Demol, N. Turk, K. Gevaert, A.G. Skirtach, M. Lamkanfi, R. Baets, "Gold Nanodome SERS platform for label-free detection of protease activity," Faraday Discussions, 205, 345-361 (2017)

[3] A. Raza, S. Clemmen, P.C. Wuytens, M. Muneeb, M. Van Daele, J. Dendooven, C. Detavernier, A. Skirtach,R. Baets, “ALD assisted nanoplasmonic slot waveguide for on-chip enhanced Raman spectroscopy", APL Photonics, 3, 116105 (2018)

[4] N. Turk, A. Raza, P.C. Wuytens, H. Demol, A. Skirtach, K. Gevaert, R. Baets, "Towards SERS-based on-chip detection of protease activity using nanoplasmonic slot waveguides", Proceedings of the 23rd Annual Symposium of the IEEE Photonics Benelux Chapter, Belgium, (2018)

[5] N. Turk, A. Raza, P.C. Wuytens, M. Van Daele, C. Detavernier, A. Skirtach, K. Gevaert, R. Baets, "Comparison of Free-Space and Waveguide-Based SERS Platforms", Nanomaterials, 9, 1404 (2019) 


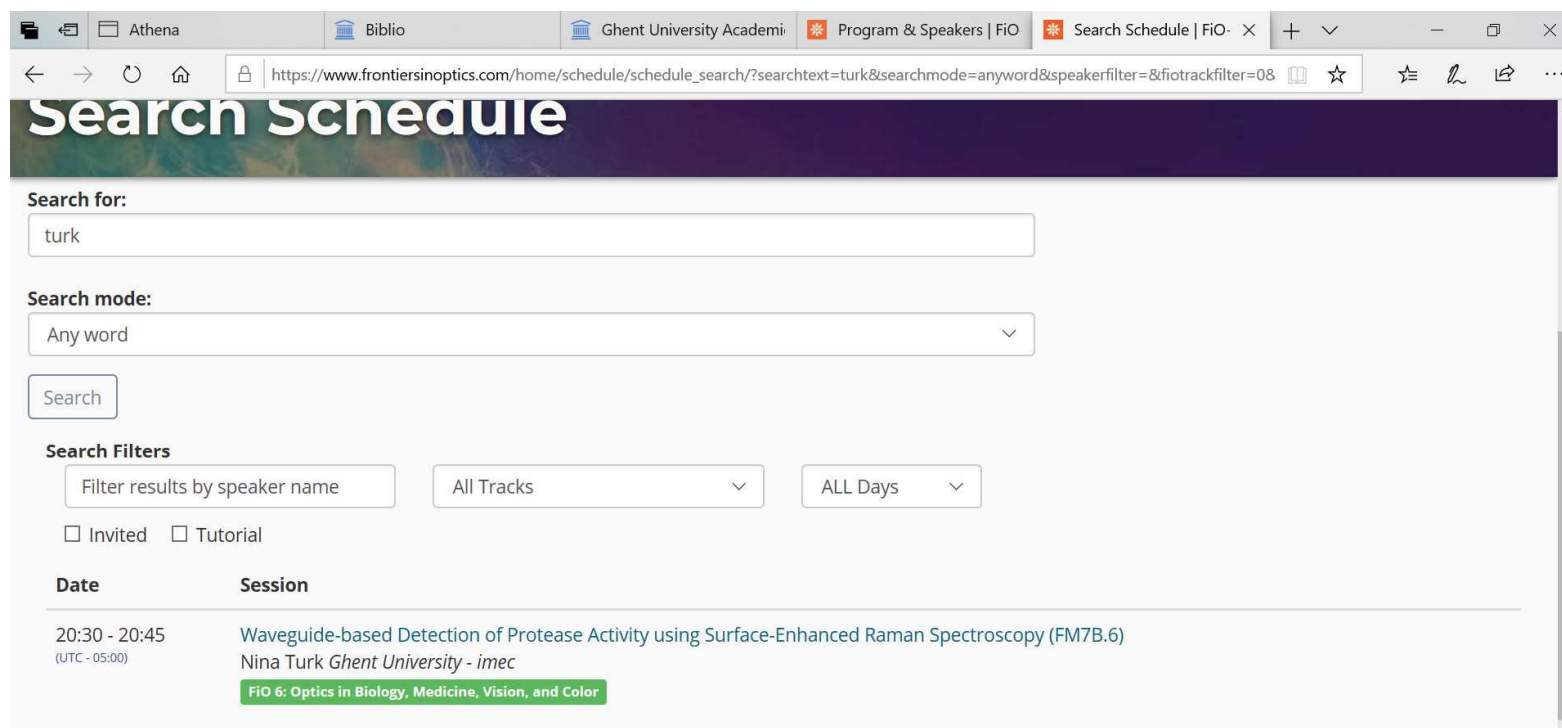

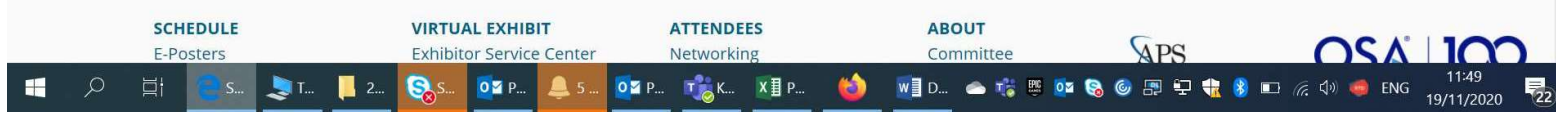

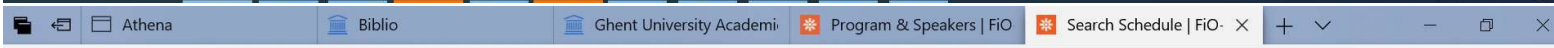

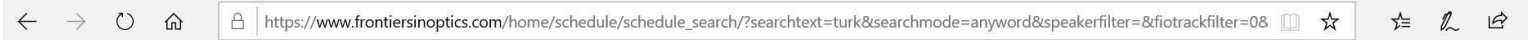
For Exhibitors \& Sponsors $\quad$ For Media $\quad$ For Speakers $\quad$ For Attendees $\quad$ Login Contact Us OSA FRONTIERS IN OPTICS LASER SCIENCE APS/DLS

Technical Conference \& Virtual Exhibits: 14 - 17 September 2020 All Sessions Held Online: Eastern Daylight Time (EDT), GMT-04:00

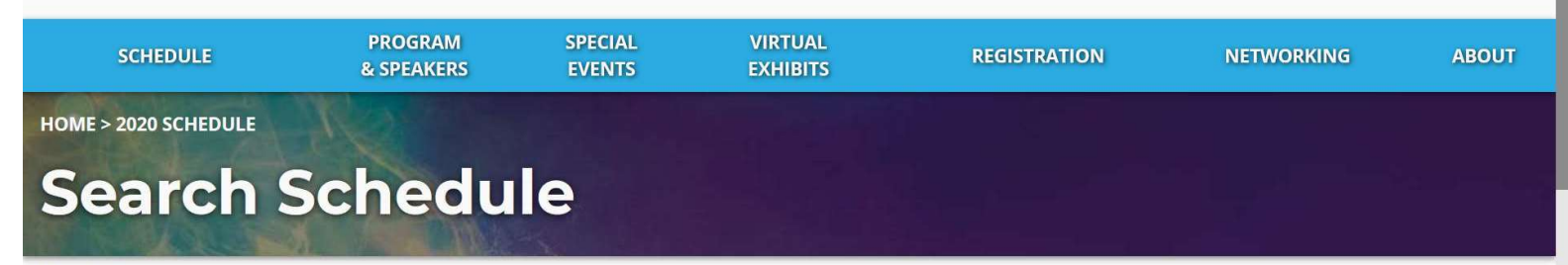

\footnotetext{
Search for:

turk

Search mode:

Any word

Search
}

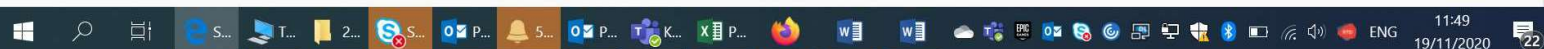

